06

\title{
Исследование переходных областей гетероструктур InAsPSb/InAs, полученных методом газофазной эпитаксии из металлоорганических соединений
}

\author{
() В.И. Васильев, Г.С. Гагис , Р.В. Левин, \\ В.И. Кучинский, А.Г. Дерягин, Д.Ю. Казанцев, Б.Я. Бер
}

Физико-технический институт им. А.Ф. Иоффре РАН, Санкт-Петербург ๑ E-mail: galina.gagis@gmail.com

Поступило в Редакцию 3 апреля 2017 г.

При исследовании полученных методом газофазной эпитаксии гетероструктур $\operatorname{InAs} \mathrm{P}_{y} \mathrm{Sb}_{1-x-y} / \mathrm{InAs}(x>0.55)$ для рассогласованных с подложкой образцов методом вторичной ионной масс-спектрометрии выявлено заметное и протяженное $(\sim 800 \mathrm{~nm})$ изменение содержания As и $\mathrm{P}(y$ до 0.12$)$ по толщине слоя, носящее экспоненциальный характер. Рассчитанное по измеренному распределению компонентов As и Р несоответствие параметров решеток было максимальным на границе эпитаксиального слоя с подложкой и уменьшалось по мере удаления от гетероинтерфейса в глубь эпитаксиального слоя.

DOI: $10.21883 /$ PJTF.2017.19.45085.16810

Многокомпонентные твердые растворы $A^{3} B^{5}$ являются перспективными материалами для устройств инфракрасной оптоэлектроники, а все более распространенным методом изготовления таких структур становится газофазная эпитаксия из металлоорганических соединений (ГФЭМОС). В настоящее время широко изучается ГФЭМОС материалов GaInAsP для фотоприемников диапазона длин волн $0.87-1.55 \mu \mathrm{m}$, при этом много внимания уделяется распределению компонентов по площади $[1,2]$. Тем не менее эпитаксиальные слои толщиной $1-3 \mu \mathrm{m}$ с высокой степенью кристаллического совершенства необходимы для создания фотоприемников, в том числе для диапазона длин волн 1.7-5 $\mu \mathrm{m}$, который охватывают материалы $A^{3} B^{5}$, содержащие сурьму. $\mathrm{B}$ частности, представляют интерес фотоприемники с активными областями на основе InAsPSb для диапазона длин волн $2-2.5 \mu \mathrm{m}$, а также 
фотоприемники для длин волн $3-5 \mu \mathrm{m}$ с активной областью InAs и барьерными слоями InAsPSb [3].

Существенное влияние на характеристики приборных структур оказывает качество гетерограниц, которое может ухудшаться за счет неконтролируемого образования переходных слоев переменного состава, содержащих дефекты несоответствия. Для широко распространенного ранее метода жидкофазной эпитаксии (ЖФЭ) проводились специальные исследования условий формирования переходных областей между слоями гетероструктуры, выводы делались на основе качества поверхности эпитаксиального слоя [4]. Процесс ЖФЭ принято рассматривать как близкий к равновесному, за счет чего становится возможным межфазное перераспределение компонентов, затрагивающее подложку, которое приводит к нерезкости границ. При ГФЭМОС образование переходных слоев принято связывать с задержкой поступления некоторых компонентов на поверхность роста в начальный момент и с характером их адсорбции. В работе [5] для образцов, полученных методом ГФЭМОС при атмосферном давлении, о переходных слоях протяженностью около $100 \mathrm{~nm}$ на гетерограницах InAsPSb/InAs выводы делались на основе исследования рентгеновских кривых дифракционного отражения. Метод вторичной ионной масс-спектрометрии (ВИМС), являющийся прямым методом исследования распределения компонентов по толщине слоя, упомянут в литературе в связи с исследованием хорошо согласованных с подложкой InP слоев GaInAsP (несоответствие параметров решетки (НПР) $\left.f=5 \cdot 10^{-4}\right)$ и сверхтонких квантовых ям [2].

Ранее нами методом ГФЭМОС были получены при пониженном давлении (76 Torr) эпитаксиальные слои твердых растворов InAsPSb толщиной $1.5-1.8 \mu \mathrm{m}$ на подложках $\operatorname{InAs}(100): \operatorname{Sn}\left(n \sim 10^{18} \mathrm{~cm}^{-3}\right)$, температура эпитаксиального роста составляла $600^{\circ} \mathrm{C}$, подробно методы получения и исследования описаны в работе [6].

В настоящей работе полученные в [6] образцы InAsPSb/InAs исследованы методом ВИМС с целью выявления возможных неоднородностей на гетерогранице, о которых можно было судить по данным рентгеновской дифрактометрии, приведенным в [6]. Номера образцов InAsPSb/InAs в настоящей работе соответствуют номерам этих же образцов в работе [6].

Во время эпитаксиального выращивания исследуемых образцов молярные потоки прекурсоров, поставляющих химические элементы: триметилиндия TMIn, триметилсурьмы TMSb и арсина $\mathrm{AsH}_{3},-$ состав-

Письма в ЖТФ, 2017, том 43, вып. 19 
Результаты исследования полученных образцов методом ВИМС

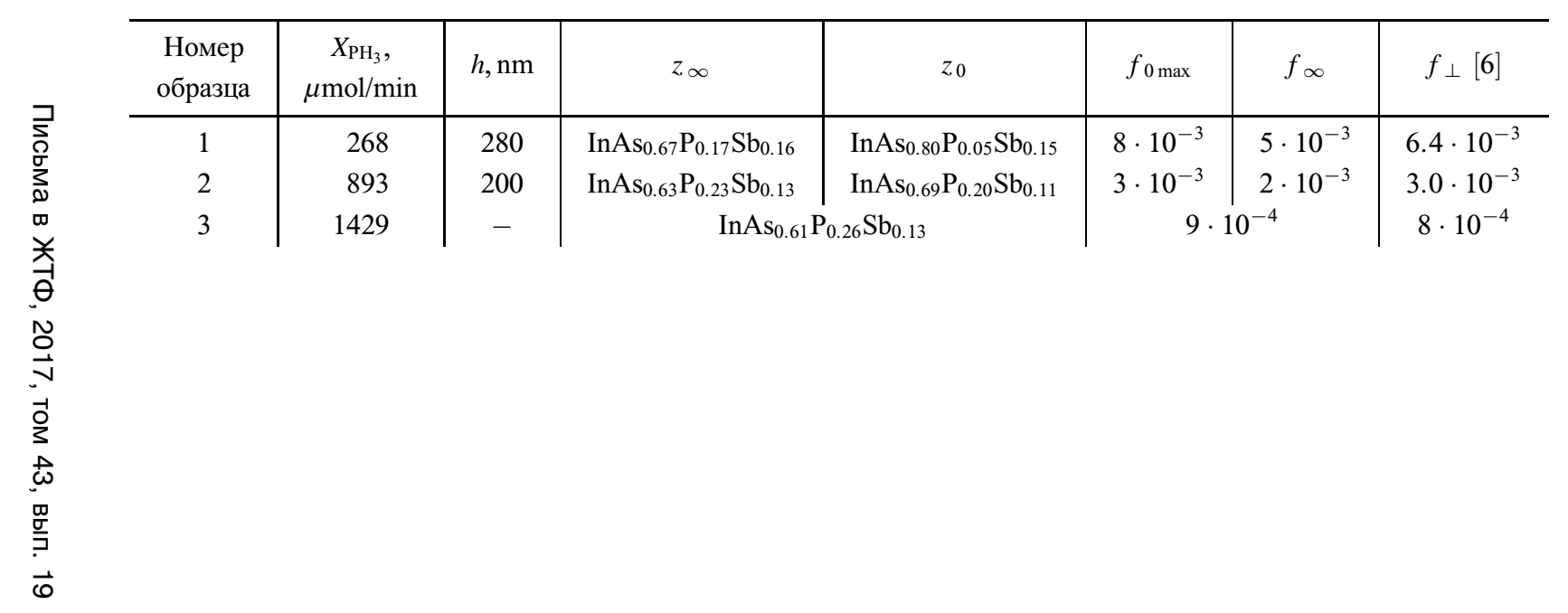


ляли $X_{\mathrm{TMIn}}=90 \mu \mathrm{mol} / \mathrm{min}, X_{\mathrm{TMSb}}=156 \mu \mathrm{mol} / \mathrm{min}, X_{\mathrm{AsH}_{3}}=51 \mu \mathrm{mol} / \mathrm{min}$ соответственно, молярные потоки фосфина $X_{\mathrm{PH}_{3}}$ для каждого из полученных образцов приведены в таблице. В процессе осаждения каждого эпитаксиального слоя, длящегося $30 \mathrm{~min}$, молярные потоки прекурсоров были постоянными, однако ВИМС у образцов № 1 и 2, выращенных при более низких $X_{\mathrm{PH}_{3}}$, чем образец № 3, показала ощутимые изменения состава по толщине слоя (см. рисунок), в то время как для образца № 3 на кривых распределения компонентов по толщине слоя переходная область у гетерограницы не может быть выявлена, поскольку наблюдаемая размытость гетерограницы $\sim 50 \mathrm{~nm}$ соответствует сглаживанию значений концентраций компонентов, обусловленному особенностями методики измерения ВИМС (рисунок, $d$ ).

Систематическая погрешность определения концентрации $z$ рассматриваемого компонента в подрешетке с помощью метода ВИМС обусловлена точностью измерения состава эталонного образца методом рентгеновского микроанализа и оценена нами как $\Delta_{s} z=0.01$. Случайная погрешность измерения состава методом ВИМС была оценена по разбросу экспериментальных значений при измерении постоянной величины и по разбросу отклонений от математических кривых, аппроксимирующих распределение компонентов по толщине слоев, и составила $\Delta_{o} z=0.005$.

Для образцов № 1 (рисунок, $a$ ) и № 2 (рисунок, $b$ ) на достаточно протяженных расстояниях (более $200 \mathrm{~nm}$ ) содержание Аs в подрешетке пятой группы существенно снижалось, а $\mathrm{P}$ - существенно увеличивалось от гетерограницы к поверхности слоя, и, как показал наш анализ, практически по всей толщине эпитаксиального слоя мольные индексы $z$ этих компонентов можно было аппроксимировать экспонентами вида

$$
z_{\exp }(d)=A_{z} \exp \left(-d / h_{z}\right)+z_{\infty}
$$

где $d$ - расстояние, отсчитываемое от гетерограницы к поверхности эпитаксиального слоя; $z_{\exp }(d)$ - экспоненциальная аппроксимация мольного индекса $z$-го компонента в растворе $\operatorname{InAs}_{x} \mathrm{P}_{y} \mathrm{Sb}_{1-x-y}$ : мышьяка $x_{\exp }(d)$ либо фосфора $y_{\exp }(d) ; A_{z}$ - масштабный множитель; $h_{z}-$ характеристическое расстояние для распределения $z$-го компонента; $z_{\infty}$ - асимптотическая величина, к которой приближается значение мольного индекса $z$-го компонента на больших расстояниях от гетерограницы $\left(d \gg h_{z}\right)$. Значение мольного индекса на гетерогранице можно

6 Письма в ЖТФ, 2017, том 43, вып. 19 

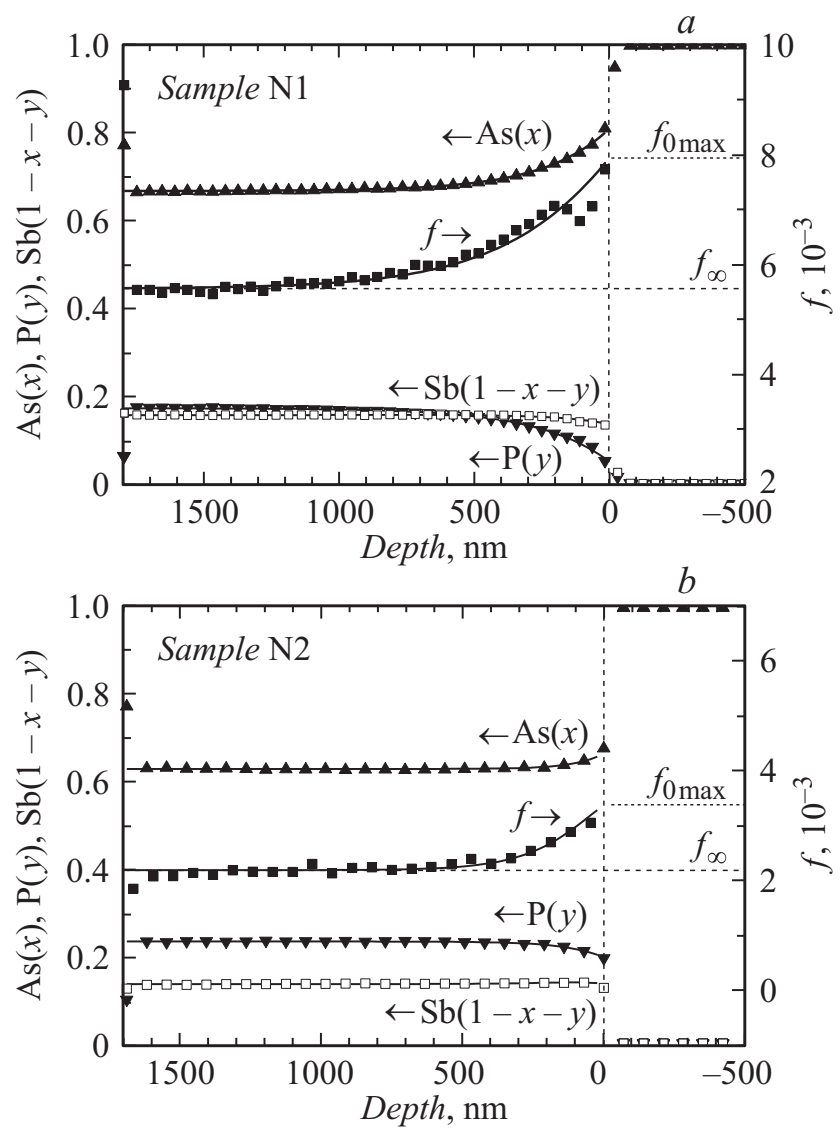

Профили распределения $(a, b)$ и фрагменты профилей распределения $(c, d)$ компонентов $\mathrm{P}$, As и $\mathrm{Sb}$ по толщине гетероструктур $\operatorname{InAs}_{x} \mathrm{P}_{y} \mathrm{Sb}_{1-x-y} / \mathrm{InAs}$, полученные методом ВИМС, а также соответствующие им расчетные зависимости $f$ для образцов № $1(a, c), 2(b), 3(d)$. Точками показаны $f_{i}$, сплошной линией $f_{\text {approx }}(d)$.

найти как $z_{0}=A_{z}+z_{\infty}$. Максимальные погрешности определения коэффициентов были следующими: $\Delta A_{z}=0.003, \Delta z_{0}=0.003, \Delta h_{z}=40 \mathrm{~nm}$. Для аппроксимации экспонентами использовались экспериментальные точки, лежащие в интервалах расстояний $d \in\left[0, h_{d}-\Delta d\right]$, где $h_{d}-$ 

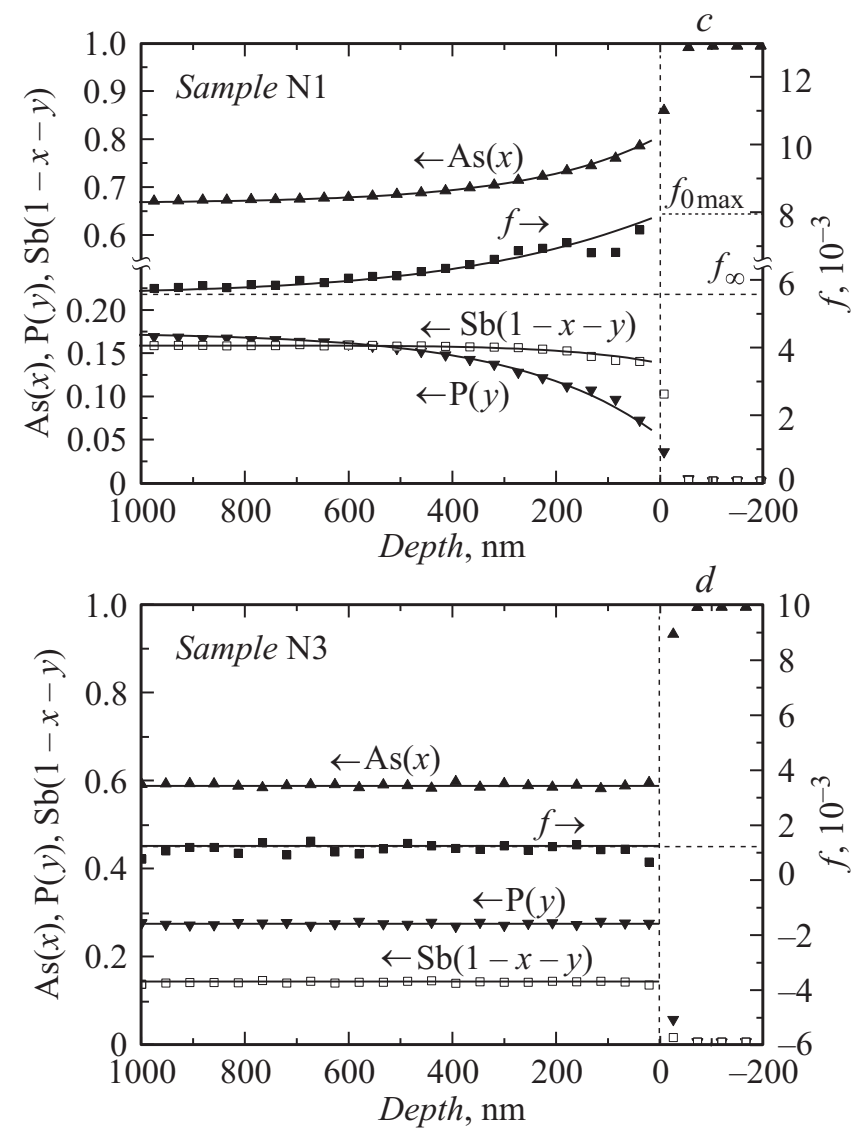

Рисунок (продолжение).

расстояние от гетерограницы $(d=0)$ до поверхности эпитаксиального слоя (толщина эпитаксиального слоя). Для образца № 1 толщина составляла $h_{d}=1800 \mathrm{~nm}$, для образца № $2-h_{d}=1700 \mathrm{~nm}$.

Экспериментально определенная с помощью ВИМС концентрация сурьмы у гетерограницы эпитаксиальных слоев образцов № 1 и 2 возрастала достаточно резко и на протяжении всего слоя практически не изменялась. Это можно объяснить довольно низкой энергией атоми-

$6^{*}$ Письма в ЖТФ, 2017, том 43, вып. 19 
зации димеров и тетрамеров сурьмы, ее малой подвижностью на поверхности роста и относительно низким давлением насыщенных паров, благодаря чему встраивание сурьмы в большей степени определяется условиями, поддерживаемыми в реакторе во время эпитаксиального роста, и в меньшей степени свойствами ростовой поверхности.

По измеренному методом ВИМС распределению компонентов мышьяка $x$ и фосфора $y$ по толщине слоя $\operatorname{InAs}_{x} \mathrm{P}_{y} \mathrm{Sb}_{1-x-y}$ была рассчитана зависимость НПР $f$ от расстояния $d$.

Зависимость параметра решетки $a$ от состава $\operatorname{InAs}_{x} \mathrm{P}_{y} \mathrm{Sb}_{1-x-y}$ определяется правилом Вегарда

$$
a(x, y)=a_{\mathrm{InAs}} x+a_{\mathrm{InP}} y+a_{\mathrm{InSb}}(1-x-y),
$$

где $a_{\mathrm{InAs}}=6.0583 \AA, a_{\mathrm{InP}}=5.8697 \AA, a_{\mathrm{InSb}}=6.4794 \AA-$ параметры решетки входящих в твердый раствор бинарных соединений [7]. Рассчитанное по составу НПР в данной работе определялось без учета деформаций как

$$
f(x, y)=\left[a(x, y)-a_{\mathrm{InAs}}\right] / a_{\mathrm{InAs}} .
$$

Нами были рассчитаны значения НПР для каждой $i$-й экспериментальной точки распределения компонентов по толщине слоя в интервалах $d \in\left[0 \mathrm{~nm}, h_{d}-50 \mathrm{~nm}\right]: f_{i}=f\left(x_{i}, y_{i}\right)$, а также для образцов № 1 и 2 - по экспоненциальным аппроксимациям мышьяка $x_{\exp }(d)$ и фосфора $y_{\exp }(d): f_{\text {approx }}(d)=f\left(x_{\exp }(d), y_{\exp }(d)\right)$. Результаты показаны на рисунке и приведены в таблице, где $h=\max \left(h_{x}, h_{y}\right)-$ характеристическое расстояние, общее для распределений фосфора и мышьяка, $f_{\infty}-$ расчетное НПР вдали от гетерограницы (на расстояниях $d \gg h), f_{0 \max }$ - максимальное значение расчетного НПР у гетерограницы на расстояниях $d<h$. Погрешность вычисления величины $f_{i}$ является погрешностью косвенных измерений и определяется погрешностями измерений концентраций мышьяка $x_{i}$ и фосфора $y_{i}$. В общем случае погрешность косвенных измерений зависит от значения измеряемой величины в каждой экспериментальной точке, но в случае зависимости (3) она является константой. Наша оценка дает величину случайной погрешности расчета НПР, связанной со случайными погрешностями измерений концентраций мышьяка $x_{i}$ и фосфора $y_{i}$ : $\Delta_{o} f=6 \cdot 10^{-4}$. Отклонения точек $f_{i}$ от $f_{\text {approx }}(d)$ лежат в пределах случайной погрешности $\Delta_{o} f$. Систематическую погрешность расчета НПР, обусловленную систематической погрешностью измерения состава, мы оцениваем как $\Delta_{s} f=1.2 \cdot 10^{-3}$. Кроме того, следует иметь

Письма в ЖТФ, 2017, том 43, вып. 19 
в виду, что рассчитанные по составу величины НПР могут расходиться с измеренными методом регистрации рентгеновских кривых дифракционного отражения значениями $f_{\perp}$, поскольку на измеренную величину влияют деформации кристалла. Для кристалла $A^{3} B^{5}$ со структурой цинковой обманки, выращенного на подложке ориентации (100), в случае полностью упругодеформированного слоя выполняется соотношение $f_{\perp} \approx 2 f_{0}$, где $f_{0}-$ равновесное НПР, такое, каким бы оно было для эпитаксиального слоя в случае отсутствия деформаций. В таблице помимо рассчитанных на гетерогранице и на большом расстоянии от нее значений $f_{0 \max }$ и $f_{\infty}$ приведены измеренные в работе [6] значения $f_{\perp}$.

Следует отметить, что у образца № 1 распределение $f_{i}$ по толщине слоя в интервале $d \in[0 \mathrm{~nm}, 250 \mathrm{~nm}]$ в пределах указанной в настоящей работе точности имеет ярко выраженный провал по сравнению с $f_{\text {approx }}(d)$. На рисунке, $c$ для образца № 1 показан увеличенный фрагмент распределения концентраций компонентов и НПР $f$ по толщине слоя, и можно видеть на расстоянии $d \approx 190 \mathrm{~nm}$ изломы для экспериментально определенных распределений фосфора $y_{i}$ и сурьмы и их отклонения от аппроксимаций $y_{\exp }(d)$ и $\left(1-x_{\exp }(d)-y_{\exp }(d)\right)$. Резонно предположить, что аналогичный излом имеется и для распределения мышьяка $x_{i}$, только при данной точности измерений он практически неразличим. Данные изломы, вероятно, связаны с изменением тенденции встраивания компонентов пятой группы за счет появления дефектов несоответствия из-за релаксации упругих напряжений, возникших за счет НПР между подложкой и эпитаксиальным слоем. Согласно нашим расчетам, для образцов № 1 и 2 у гетерограницы $(d=0)$ значение НПР выше, чем на значительном расстоянии от нее $\left(f_{0 \max }>f_{\infty}\right)$. Связать переходные слои у гетерограниц с неравномерностью поступления компонентов в реактор, как это было сделано в работе [5], в данном случае мы не можем, поскольку эпитаксиальный рост проводился при пониженном давлении, которое способствует быстрой замене газовой смеси, что должно благоприятно сказываться на резкости гетерограниц [8]. К тому же, для образца № 3, полученного нами при тех же условиях, что и образцы № 1 и 2, граница была достаточно резкой, а ВИМС показывает практически постоянный состав по толщине всего эпитаксиального слоя (рисунок, $d$ ).

Анализ полученных данных позволяет предположить, что в том случае, когда удается подобрать молярные потоки прекурсоров таким образом, чтобы из них осаждался твердый раствор, хорошо

Письма в ЖТФ, 2017, том 43, вып. 19 
согласованный с подложкой, распределение компонентов по толщине слоя получается достаточно однородным. Иначе за счет НПР между подложкой InAs и растущим слоем InAsPSb возникает тетрагональная деформация, которая существенно затрудняет встраивание атомов фосфора, в результате чего в подрешетку пятой группы встраивается больше мышьяка. Поскольку фосфор присутствует в газовой фазе, какое-то его количество будет встраиваться в твердую фазу, несмотря на препятствующие факторы, в результате будут меняться свойства поверхности роста и встраивание фосфора в последующий монослой, что должно соответствовать экспоненциальному закону распределения, который мы наблюдали экспериментально.

Данные настоящей работы дополнят сведения о процессах, имеющих место при ГФЭМОС, что в дальнейшем поможет планированию режимов эпитаксиального выращивания для получения гетероструктур с высоким кристаллическим совершенством.

ВИМС измерения проводились с использованием оборудования ЦКП „Материаловедение и диагностика в передовых технологиях“ (ФТИ им. А.Ф. Иоффе), поддерживаемом Минобрнауки.

\section{Список литературы}

[1] Feron O., Sugiyama M., Asawamethapant W., Futukuchi N., Feurprier Y., Nakano Y., Shimogaki Y. // Appl. Surf. Sci. 2000. V. 159-160. P. 318-327.

[2] Razeghi M. MOCVD challenge. Survey of GaInAsP-InP \& GaInAsP-GaAs for photonic and electronic device applications. 2nd. Taylor and Francis/CRC Press, 2010. $773 \mathrm{p}$.

[3] Закгейм А.Л., Ильинская Н.Д., Карандашев С.А., Лавров А.А., Матвеев Б.А., Ременный М.А., Стусь Н.М., Усикова А.А., Черняков А.Е. // ФТП. 2017. Т. 51. B. 2. C. 269-275.

[4] Bolkhovityanov Yu.B., Chikichev S.I. // Crystal. Res. Tecnol. 1983. V. 18. N 7. P. 847-857.

[5] Романов В.В., Байдакова М.В., Моисеев К.Д. // ФТП. 2014. Т. 48. В. 6. C. $753-758$.

[6] Васильев В.И., Гагис Г.С., Левин Р.В., Дерягин А.Г., Кучинский В.И., Пушный Б.В. // Письма в ЖТФ. 2012. Т. 38. В. 9, С. 23-30.

[7] Vurgaftman I., Meyer J.R., Ram-Mohan L.R. // J. Appl. Phys. 2001. V. 89. N 11. P. 5815-5875.

[8] Duchemin J.R., Hirtz J.P., Razeghi M, Bonnet M., Hersee S.D. // J. Cryst. Growth. 1981. V. 55. P. 64-73. 